\title{
PENALIZED INTEGRATIVE ANALYSIS UNDER THE ACCELERATED FAILURE TIME MODEL
}

\author{
Qingzhao Zhang ${ }^{1}$, Sanguo Zhang ${ }^{1}$, Jin Liu $^{2}$, Jian Huang ${ }^{3}$ and Shuangge Ma ${ }^{4}$ \\ ${ }^{1}$ University of Chinese Academy of Sciences, ${ }^{2}$ Duke-NUS School of Medicine, \\ ${ }^{3}$ University of Iowa and ${ }^{4}$ Yale University
}

\begin{abstract}
For survival data with high-dimensional covariates, results generated in the analysis of a single dataset are often unsatisfactory because of the small sample size. Integrative analysis pools raw data from multiple independent studies with comparable designs, effectively increases sample size, and has better performance than meta-analysis and single-dataset analysis. In this study, we conduct integrative analysis of survival data under the accelerated failure time (AFT) model. The sparsity structures of multiple datasets are described using homogeneity and heterogeneity models. For variable selection under the homogeneity model, we adopt group penalization approaches; for variable selection under the heterogeneity model, we use composite penalization and sparse group penalization approaches. As a major advancement from existing studies, the asymptotic selection and estimation properties are rigorously established. Simulation study is conducted to compare different penalization methods and against alternatives. We also analyze four lung cancer prognosis datasets with gene expression measurements.
\end{abstract}

Key words and phrases: Consistency properties, homogeneity and heterogeneity models, integrative analysis, penalized selection.

\section{Introduction}

In survival studies, data with high-dimensional covariates are commonly encountered. A lung cancer prognosis study with gene expression measurements is presented in this article, and more are available in the literature. With such "large $p$, small $n$ " data, results generated in the analysis of a single dataset are often unsatisfactory because of the small sample size (Guerra and Goldstein (200.9); Lilu et al. (2013); Ma et al. (2011b)). For outcomes of common interest, there are often multiple independent studies with comparable designs. This makes it possible to pool multiple datasets, increase sample size, and improve over single-dataset analysis. As a family of multi-dataset analysis methods, integrative analysis methods pool and analyze raw data from multiple studies, and they outperform classic meta-analysis methods that analyze multiple datasets separately before combining summary statistics. 
In this article, we conduct an integrative analysis of multiple independent survival datasets under the accelerated failure time (AFT) model. The analysis goal is to identify, given a large number of measured covariates, important markers associated with survival. In this we adopt penalization, the choice of many high-dimensional studies. A large number of penalization methods have been developed for single-dataset analysis. However because of the multi-dataset settings and heterogeneity across datasets, they are not applicable to integrative analysis. The sparsity structures of multiple datasets can be described using homogeneity and heterogeneity models. Different models demand marker selection with different properties and hence different methods. This makes integrative analysis even more complicated. Penalization methods for integrative analysis have been developed (Liu et al. (2013); Ma et al. (2011b)), but not in a systematic manner.

This study makes advances from single-dataset analysis and meta-analysis by conducting integrative analysis of multiple heterogeneous datasets. It conducts more systematic investigation than existing integrative analysis studies such as Lill et al (2013); Ma et al (2011b). And more importantly, it rigorously establishes the selection and estimation properties not previously examined. The theoretical development is nontrivial because of data complexity, model settings, and penalties. Properties of composite penalization and sparse group penalization have not been studied for single-dataset analysis under the AFT model. Thus our study can provide insights for single-dataset penalization methods. Our study also advances from existing studies by conducting systematic simulations and direct comparisons of multiple methods.

Data and model settings are described in Section 2. Penalized integrative analyses under homogeneity and heterogeneity models are investigated in Sections 3 and 4, respectively. Our numerical study is reported on in Section 5. The article concludes with discussion in Section 6. Technical details and additional analysis results are in the Appendix.

\section{Integrative Analysis under AFT Model}

Consider the integrative analysis of survival data from $M$ independent studies. In study $m(=1, \ldots, M)$ with $n_{m}$ iid subjects, let $\boldsymbol{T}^{m}=\left(T_{1}^{m}, \ldots, T_{n_{m}}^{m}\right)^{\top}$ be the logarithm of failure times and $\boldsymbol{X}^{m} \in R^{n_{m} \times p_{m}}$ be the predictor matrix. Assume the AFT model

$$
\boldsymbol{T}^{m}=\boldsymbol{X}^{m} \boldsymbol{\beta}^{m}+\boldsymbol{\epsilon}^{m},
$$

where $\boldsymbol{\beta}^{m}$ is the vector of regression coefficients, and $\boldsymbol{\epsilon}^{m}$ is the vector of random errors. With proper normalization, the intercept term has been omitted. Assume that all datasets measure the same set of covariates. Then $p_{1}=\cdots=p_{M}=p$. When different datasets have mismatched covariate sets, a rescaling approach 
(Ma, Huang and Song (2017a); Lill et all (2013)) can be adopted. The proposed approaches are then applicable with minor modifications.

Let $\boldsymbol{\beta}=\left(\boldsymbol{\beta}^{1}, \ldots, \boldsymbol{\beta}^{M}\right)=\left(\boldsymbol{\beta}_{1}, \ldots, \boldsymbol{\beta}_{p}\right)^{\top}$, where $\boldsymbol{\beta}_{j}=\left(\beta_{j}^{1}, \ldots, \beta_{j}^{M}\right)^{\top}$ consists of the coefficients of variable $j$ in all $M$ datasets. Write $\boldsymbol{\beta}=\left(\beta_{i j}\right)_{p \times M}$ with its true value $\boldsymbol{\beta}^{*}$, where $\beta_{i j}=\beta_{i}^{j}$. With heterogeneity across datasets, $\beta_{j}^{m}$ is not necessarily equal to $\beta_{j}^{k}$ for $m \neq k$. Under right censoring, one observes $\left(\boldsymbol{Y}^{m}, \boldsymbol{\delta}^{m}, \boldsymbol{X}^{m}\right)$ with $\boldsymbol{Y}^{m}=\boldsymbol{T}^{m} \wedge \boldsymbol{C}^{m}$, where $\boldsymbol{C}^{m}$ is the vector of log censoring times, and $\boldsymbol{\delta}^{m}=1\left\{\boldsymbol{T}^{m} \leq \boldsymbol{C}^{m}\right\}$.

When the distribution of random errors is unknown, there are multiple estimation approaches (Ying (109.93)). We adopt the weighted least squares (LS) approach (Stute (11993)) that has the lowest computational cost and is desirable with high-dimensional data. Let $\hat{F}^{m}$ be the Kaplan-Meier estimator of the distribution function $F^{m}$ of $T^{m}$. Let $Y_{(1)}^{m} \leq \cdots \leq Y_{\left(n_{m}\right)}^{m}$ be the order statistics of $Y_{i}^{m}$ 's. $\hat{F}^{m}$ can be written as $\hat{F}^{m}(y)=\sum_{i=1}^{n_{m}} \omega_{i}^{m} 1\left\{Y_{i}^{m} \leq y\right\}$, where $\omega_{i}^{m}$ 's are expressed as

$$
\omega_{1}^{m}=\frac{\delta_{(1)}^{m}}{n_{m}} \quad \text { and } \quad \omega_{i}^{m}=\frac{\delta_{(i)}^{m}}{n_{m}-i+1} \prod_{j=1}^{i-1}\left(\frac{n_{m}-j}{n_{m}-j+1}\right)^{\delta_{(j)}^{m}}, \quad i=2, \ldots, n_{m} .
$$

Here $\delta_{(1)}^{m}, \ldots, \delta_{\left(n_{m}\right)}^{m}$ are the associated censoring indicators of the ordered $Y_{i}^{m}$ 's. Write $W_{m}=\operatorname{diag}\left\{n_{m} \omega_{1}^{m}, \ldots, n_{m} \omega_{n_{m}}^{m}\right\}$. Then for the $M$ datasets combined, the weighted LS approach is to minimize

$$
\tilde{L}(\boldsymbol{\beta})=\frac{1}{2 n} \sum_{m=1}^{M}\left(\boldsymbol{Y}^{m}-\boldsymbol{X}^{m} \boldsymbol{\beta}^{m}\right)^{\top} W_{m}\left(\boldsymbol{Y}^{m}-\boldsymbol{X}^{m} \boldsymbol{\beta}^{m}\right),
$$

in which the components of $\boldsymbol{Y}^{m}$ and $\boldsymbol{X}^{m}$ need to be sorted. We need an assumption.

Condition 1. For all vector $\boldsymbol{\nu}$ with $\|\boldsymbol{\nu}\|_{2}=1$ and any $t \geq 0, P\left(\left|\boldsymbol{\nu}^{\top} \boldsymbol{\epsilon}^{m}\right| \geq t\right) \leq$ $2 \exp \left(-t^{2} / 2 \sigma_{m}^{2}\right)$, and $\boldsymbol{\epsilon}^{m}$ is independent of $W_{m}$.

The total sample size is $n=\sum_{m=1}^{M} n_{m}$. The important predictor index sets of $M$ datasets are labeled as $S_{1}, \ldots, S_{M}$, respectively. Then $S=\bigcup_{m=1}^{M} S_{m}$ denotes the important set. Let $S^{c}$ and $|S|$ denote the complement and cardinality of set $S$, respectively. Let $\mathcal{A}=\left\{(i, j): \beta_{i j}^{*} \neq 0\right\}$ and $\mathcal{B}=\{(i, j): i \in S, j=1, \ldots, M\}$. Let $\boldsymbol{\beta}_{\mathcal{A}}$ and $\boldsymbol{\beta}_{\mathcal{B}}$ denote the components of $\boldsymbol{\beta}$ indexed by $\mathcal{A}$ and $\mathcal{B}$, respectively. For a $p \times 1$ vector $v$ and index set $I \subset\{1, \ldots, p\}$, let $v_{I}$ denote the components of $v$ indexed by $I$, and let $\boldsymbol{X}^{m, i}$ denotes the transposition of the $i$ th row of $\boldsymbol{X}^{m}$. Then for any index set $I \subset\{1, \ldots, p\}, \boldsymbol{X}_{I}^{m}=\left(\boldsymbol{X}_{I}^{m, 1}, \ldots, \boldsymbol{X}_{I}^{m, n_{m}}\right)^{\top}$.

\subsection{Homogeneity and heterogeneity models}

The sparsity structure of $\boldsymbol{\beta}$ can be described using homogeneity and heterogeneity models. Under the homogeneity model, $\boldsymbol{\beta}^{m}$ 's have the same sparsity 
structure, so $I\left(\beta_{j}^{m}=0\right)=I\left(\beta_{j}^{k}=0\right)$ for all $(m, k, j)$ 's. The intuition is that if the $M$ datasets are "close enough", then the same set of markers should be identified in all datasets. Under this model, we only need to determine whether a covariate is important or not. With the (sometimes great) differences across datasets, the homogeneity model may be too restrictive. The heterogeneity model allows different datasets to have different sparsity structures. Under this model, we need to determine whether a covariate is associated with any response at all and, for an important covariate, we need to determine in which datasets it is important. That is, a two-level selection is needed.

\section{Integrative Analysis under the Homogeneity Model}

Under this model, the required one-level selection can be achieved using group penalization. In terms of formulation and computation, the development of group penalization methods in integrative analysis share some similarity with that in single-dataset analysis (Bühlmann and van de (Geer (2011)), but with the significantly different data settings and adoption of the AFT model, the theoretical development has significant differences.

\subsection{Group LASSO}

Consider the group LASSO penalized objective function

$$
L(\boldsymbol{\beta})=\frac{1}{2 n} \sum_{m=1}^{M}\left(\boldsymbol{Y}^{m}-\boldsymbol{X}^{m} \boldsymbol{\beta}^{m}\right)^{\top} W_{m}\left(\boldsymbol{Y}^{m}-\boldsymbol{X}^{m} \boldsymbol{\beta}^{m}\right)+\lambda \sum_{j=1}^{p}\left\|\boldsymbol{\beta}_{j}\right\|_{2},
$$

where $\lambda$ is the tuning parameter and $\left\|\boldsymbol{\beta}_{j}\right\|_{2}=\left[\left(\beta_{j}^{1}\right)^{2}+\cdots+\left(\beta_{j}^{M}\right)^{2}\right]^{1 / 2}$.

For set $S$, define the estimate $\hat{\boldsymbol{\beta}}_{\mathcal{B}}=\left(\hat{\boldsymbol{\beta}}_{S}^{1}, \ldots, \hat{\boldsymbol{\beta}}_{S}^{M}\right)$ as

$$
\hat{\boldsymbol{\beta}}_{\mathcal{B}}=\underset{\boldsymbol{\beta}_{\mathcal{B}}}{\operatorname{argmin}}\left\{\frac{1}{2 n} \sum_{m=1}^{M}\left(\boldsymbol{Y}^{m}-\boldsymbol{X}_{S}^{m} \boldsymbol{\beta}_{S}^{m}\right)^{\top} W_{m}\left(\boldsymbol{Y}^{m}-\boldsymbol{X}_{S}^{m} \boldsymbol{\beta}_{S}^{m}\right)+\lambda \sum_{j \in S}\left\|\boldsymbol{\beta}_{j}\right\|_{2}\right\} .
$$

For the group LASSO to be able to consistently identify the true sparsity structure, one needs a local solution $\hat{\boldsymbol{\beta}}^{\text {glasso }}=\left\{\hat{\boldsymbol{\beta}}_{\mathcal{B}}^{\text {glasso }}, \hat{\boldsymbol{\beta}}_{\mathcal{B}^{c}}^{\text {glasso }}\right\}$ for (‥D), where $\hat{\boldsymbol{\beta}}_{\mathcal{B}}^{\text {glasso }}=\hat{\boldsymbol{\beta}}_{\mathcal{B}}$ and $\hat{\boldsymbol{\beta}}_{\mathcal{B}^{c}}^{\text {glasso }}=0$. Take

$$
\begin{aligned}
& \bar{\rho}_{2}^{m}=\lambda_{\max }\left\{n_{m}^{-1} \boldsymbol{X}_{S}^{m \top} W_{m}^{2} \boldsymbol{X}_{S}^{m}\right\}, \underline{\rho}_{1}^{m}=\lambda_{\min }\left\{n_{m}^{-1} \boldsymbol{X}_{S}^{m \top} W_{m} \boldsymbol{X}_{S}^{m}\right\}, \\
& \Lambda_{m}=\max _{j}\left\{n_{m}^{-1} \boldsymbol{X}_{j}^{m \top} W_{m}{ }^{2} \boldsymbol{X}_{j}^{m}\right\}, \psi_{m}=\left\|\boldsymbol{X}_{S^{c}}^{m \top} W_{m} \boldsymbol{X}_{S}^{m}\left(\boldsymbol{X}_{S}^{m \top} W_{m} \boldsymbol{X}_{S}^{m}\right)^{-1}\right\|_{\infty} .
\end{aligned}
$$

Theorem 1. Let Condition 1 hold.

1. There exists a local minimizer $\hat{\boldsymbol{\beta}}_{\mathcal{B}}$ of (․2) such that 


$$
\begin{aligned}
& \operatorname{Pr}\left\{\left\|\hat{\boldsymbol{\beta}}_{S}^{m}-\boldsymbol{\beta}_{S}^{m *}\right\|_{2} \leq \lambda \sqrt{|S|} \frac{4}{\underline{\rho}_{1}^{m}} \frac{n}{n_{m}}, m=1, \ldots, M\right\} \\
& \geq 1-\sum_{m=1}^{M} \exp \left(-\frac{\lambda^{2}|S| n^{2}}{2 \sigma_{m}^{2} \bar{\rho}_{2}^{m} n_{m}}\right) .
\end{aligned}
$$

2. If the ir-representable conditions $\psi_{m} \leq D_{m}<1$ hold. Then $\hat{\boldsymbol{\beta}}^{\text {glasso }}=\left\{\hat{\boldsymbol{\beta}}_{\mathcal{B}}^{\text {glasso }}\right.$, $\left.\hat{\boldsymbol{\beta}}_{\mathcal{B}^{c}}^{\text {glasso }}\right\}$ with $\hat{\boldsymbol{\beta}}_{\mathcal{B}}^{\text {glasso }}=\hat{\boldsymbol{\beta}}_{\mathcal{B}}, \hat{\boldsymbol{\beta}}_{\mathcal{B}^{c}}^{\text {glasso }}=0$ is a local minimizer of (B.D) with probability at least

$$
1-\sum_{m=1}^{M} \exp \left(-\frac{\lambda^{2}|S| n^{2}}{2 \sigma_{m}^{2} \bar{\rho}_{2}^{m} n_{m}}\right)-2 p \sum_{m=1}^{M} \exp \left\{-\frac{n^{2} \lambda^{2}\left(1-D_{m}\right)^{2}}{2 n_{m} \Lambda_{m} \sigma_{m}^{2}\left(1+D_{m}\right)^{2}}\right\} .
$$

In single-dataset analysis, Zhao and Yu (2006) and followup studies establish selection consistency under the ir-representable condition. Under a similar condition for individual datasets, integrative analysis also has selection consistency.

With the probability bounds in Theorem 1, we can obtain a more straightforward understanding of the penalized estimators.

Corollary 1. Suppose that for $m=1, \ldots, M, \rho_{1}^{m}, \bar{\rho}_{2}^{m}$, and $\Lambda_{m}$ are bounded away from zero and infinity. Assume that $n / n_{m}=\bar{O}(1),|S| \ll n$, and $\log p=O\left(n^{\alpha}\right)$ with $\alpha<1$. Under Condition 1 and the ir-representable conditions in Theorem 1, if $|S|^{-1 / 2} \min _{j \in S}\left\|\boldsymbol{\beta}_{j}^{*}\right\|_{2} \gg \lambda \gg n^{(\alpha-1) / 2}$, then the group LASSO can identify the true sparsity structure and $\left\|\hat{\boldsymbol{\beta}}_{S}^{m}-\boldsymbol{\beta}_{S}^{m *}\right\|_{2}=O_{p}(\lambda \sqrt{|S|}), m=1, \ldots, M$.

Remark 1. It is known that in single-dataset analysis the group LASSO is group selection consistent under some variants of the ir-representable condition, see Huang, Breheny, and Ma (2012) and others for reference. Similar conditions are needed in the integrative analysis with group LASSO. The conditions in Corollary 1 on $\rho_{1}^{m}, \bar{\rho}_{2}^{m}$, and $\Lambda_{m}$ are on the design matrixes and censoring probabilities. Corollary 1 shows that even when the group LASSO can identify the true sparsity structure, $\lambda$ should be much large than $n^{-1 / 2}$, leading to $\left\|\hat{\boldsymbol{\beta}}_{S}^{m}-\boldsymbol{\beta}_{S}^{m *}\right\|_{2} \gg \sqrt{|S| / n}$.

\subsection{Concave 2-norm group selection}

Consider penalization built on concave penalties. Notable examples of concave penalty include SCAD (Fan and Lil (2001)) and MCP (Zhang (2010)). For $t \geq 0$, the SCAD penalty has first order derivative $p_{\lambda}^{\prime}(t)=\lambda\{I(t \leq \lambda)$ $\left.+\left[(a \lambda-t)_{+} /((a-1) \lambda)\right] I(t>\lambda)\right\}$, for some $a>2$. The MCP has derivative $p_{\lambda}^{\prime}(t)=\lambda(1-t /(a \lambda))_{+}$, for some $a>1$. Consider the objective function

$$
L(\boldsymbol{\beta})=\frac{1}{2 n} \sum_{m=1}^{M}\left(\boldsymbol{Y}^{m}-\boldsymbol{X}^{m} \boldsymbol{\beta}^{m}\right)^{\top} W_{m}\left(\boldsymbol{Y}^{m}-\boldsymbol{X}^{m} \boldsymbol{\beta}^{m}\right)+\sum_{j=1}^{p} p_{\lambda}\left(\left\|\boldsymbol{\beta}_{j}\right\|_{2}\right),
$$


where the penalty $p_{\lambda}(\cdot)$ satisfies the following.

Condition 2. $\lambda^{-1} p_{\lambda}(t)$ is concave in $t \in[0, \infty)$ with a continuous derivative $\lambda^{-1} p_{\lambda}^{\prime}(t)$ satisfying $\lambda^{-1} p_{\lambda}^{\prime}(0+) \in(0, \infty)$. In addition, $\lambda^{-1} p_{\lambda}^{\prime}(t)$ is increasing in $\lambda \in(0,+\infty)$, and $\lambda^{-1} p_{\lambda}^{\prime}(0+)$ is independent of $\lambda$.

Condition 3. $\theta=\inf \left\{t / \lambda: \lambda^{-1} p_{\lambda}^{\prime}(t)=0, t \geq 0\right\}$ is bounded.

Remark 2. Condition 2 was also considered by Fan and Lv (2011). LASSO, $\mathrm{SCAD}$, and MCP all satisfy this condition. Condition 3 is added to guarantee unbiasedness. LASSO does not satisfy Condition 3 since $\lambda^{-1} p_{\lambda}^{\prime}(t)=1$ leads to $\theta=\infty$, while SCAD and MCP satisfy with $\theta=a$. Another approach is the 2-norm group bridge (Ma et al. (2012)). Under certain conditions, its selection consistency was established in Ma, Huang and Song (201।a). The bridge penalty does not satisfy Condition 3 and needs to be separately investigated.

Consider the properties of concave 2-norm group penalization. Define the oracle estimator as $\hat{\boldsymbol{\beta}}^{\text {oracle }}=\left\{\hat{\boldsymbol{\beta}}_{\mathcal{B}}^{\text {oracle }}, \hat{\boldsymbol{\beta}}_{\mathcal{B}^{c}}^{\text {oracle }}\right\}$ with $\hat{\boldsymbol{\beta}}_{\mathcal{B}}^{\text {oracle }}=\tilde{\boldsymbol{\beta}}_{\mathcal{B}}$ and $\hat{\boldsymbol{\beta}}_{\mathcal{B}^{c}}^{\text {oracle }}=0$, where

$$
\tilde{\boldsymbol{\beta}}_{\mathcal{B}}=\underset{\boldsymbol{\beta}_{S}}{\operatorname{argmin}}\left\{\frac{1}{2 n} \sum_{m=1}^{M}\left(\boldsymbol{Y}^{m}-\boldsymbol{X}_{S}^{m} \boldsymbol{\beta}_{S}^{m}\right)^{\top} W_{m}\left(\boldsymbol{Y}^{m}-\boldsymbol{X}_{S}^{m} \boldsymbol{\beta}_{S}^{m}\right)\right\} .
$$

Theorem 2. Let Conditions 1-3 hold.

1. For any $R_{m}<\sqrt{n_{m} /|S|}$, we have

$$
\operatorname{Pr}\left(\left\|\tilde{\boldsymbol{\beta}}_{S}^{m}-\boldsymbol{\beta}_{S}^{m *}\right\| \leq \sqrt{\frac{|S|}{n_{m}}} R_{m}, m=1, \ldots, M\right) \geq 1-\sum_{m=1}^{M} \exp \left\{-R_{m}^{2} \frac{|S|\left(\underline{\rho}_{1}^{m}\right)^{2}}{8 \bar{\rho}_{2}^{m} \sigma_{m}^{2}}\right\} .
$$

2. Suppose $\lambda<\min _{j \in S}\left\|\boldsymbol{\beta}_{j}^{*}\right\|_{2} / 2 \theta$ and $R_{m}^{\dagger} \leq\left[\min _{j \in S}\left\|\boldsymbol{\beta}_{j}^{*}\right\|_{2} / 2 \sqrt{M}\right] \sqrt{n_{m} /|S|}$.

Then with probability at least

$$
1-\sum_{m=1}^{M} \exp \left\{-\frac{|S|\left(\underline{\rho}_{1}^{m}\right)^{2}}{8 \bar{\rho}_{2}^{m} \sigma_{m}^{2}} R_{m}^{\dagger 2}\right\}-2 p \sum_{m=1}^{M} \exp \left\{-\frac{n^{2} p_{\lambda}^{\prime 2}(0+)}{2 n_{m} \Lambda_{m} \sigma_{m}^{2}\left(1+\psi_{m}\right)^{2}}\right\},
$$

$\hat{\boldsymbol{\beta}}^{\text {oracle }}$ is a local minimizer of (B.3).

Corollary 2. Suppose that for $m=1, \ldots, M, \underline{\rho}_{1}^{m}, \bar{\rho}_{2}^{m}$ and $\Lambda_{m}$ are bounded away from zero and infinity, $n / n_{m}=O(1),|S| \ll n, \log p=O\left(n^{\alpha}\right)$ with $\alpha<1$, and $\psi_{m}=O\left(n^{\alpha_{1}}\right)$ with $\alpha_{1} \in[0,1 / 2)$. Under Conditions $1-3$, if $\lambda<\min _{j \in S}\left\|\boldsymbol{\beta}_{j}^{*}\right\| / 2 \theta$ and $\lambda \gg n^{(\alpha-1) / 2+\alpha_{1}}$, then the concave 2-norm group selection can identify the true sparsity structure and $\left\|\hat{\boldsymbol{\beta}}_{S}^{m}-\boldsymbol{\beta}_{S}^{m *}\right\|_{2}=O_{p}\left(\sqrt{|S| / n_{m}}\right)$. 
Remark 3. When the concave penalty is used, the upper bound of $\psi_{m}$ can grow to $\infty$ at rate $O\left(n^{\alpha_{1}}\right)$. In contrast, the group LASSO needs the ir-representable conditions. Moreover, the group LASSO yields a larger bias than the concave 2-norm group selection.

\section{Integrative Analysis under the Heterogeneity Model}

Under this model, two-level selection is needed and can be achieved using composite penalization and sparse group penalization. Properties of composite penalization have been studied in single-dataset analysis, however, under much simpler data and model settings. For sparse group penalization built on concave penalties, properties have not been established for single-dataset analysis.

Define the oracle estimator $\check{\boldsymbol{\beta}}=\left\{\check{\boldsymbol{\beta}}_{\mathcal{A}}, 0\right\}$ where

$$
\check{\boldsymbol{\beta}}_{\mathcal{A}}=\underset{\boldsymbol{\beta}_{\mathcal{A}}}{\operatorname{argmin}}\left\{\frac{1}{2 n} \sum_{m=1}^{M}\left(\boldsymbol{Y}^{m}-\boldsymbol{X}_{S_{m}}^{m} \boldsymbol{\beta}_{S_{m}}^{m}\right)^{\top} W_{m}\left(\boldsymbol{Y}^{m}-\boldsymbol{X}_{S_{m}}^{m} \boldsymbol{\beta}_{S_{m}}^{m}\right)\right\} .
$$

Let $\bar{\rho}_{2}^{* m}=\lambda_{\max }\left\{n_{m}^{-1} \boldsymbol{X}_{S_{m}}^{m \top} W_{m}^{2} \boldsymbol{X}_{S_{m}}^{m}\right\}, \underline{\rho}_{1}^{* m}=\lambda_{\min }\left\{n_{m}^{-1} \boldsymbol{X}_{S_{m}}^{m \top} W_{m} \boldsymbol{X}_{S_{m}}^{m}\right\}$, and $\psi_{m}^{*}=\left\|\boldsymbol{X}_{S_{m}^{c}}^{m \top} W_{m} \boldsymbol{X}_{S_{m}}^{m}\left(\boldsymbol{X}_{S_{m}}^{m \top} W_{m} \boldsymbol{X}_{S_{m}}^{m}\right)^{-1}\right\|_{\infty}$.

Theorem 3. Under Conditions 1-3, we have

$$
\begin{aligned}
& \operatorname{Pr}\left\{\left\|\check{\boldsymbol{\beta}}_{S_{m}}^{m}-\boldsymbol{\beta}_{S_{m}}^{m}{ }^{*}\right\|_{2} \leq \sqrt{\frac{\left|S_{m}\right|}{n_{m}}} C_{m}, m=1, \ldots, M\right\} \\
& \geq 1-\sum_{m=1}^{M} \exp \left\{-C_{m}^{2} \frac{\left|S_{m}\right|\left(\underline{\rho}_{1}^{* m}\right)^{2}}{8 \bar{\rho}_{2}^{* m} \sigma_{m}^{2}}\right\}
\end{aligned}
$$

with $C_{m}<\sqrt{n_{m} /\left|S_{m}\right|}$.

Corollary 3. Suppose that for $m=1, \ldots, M, \underline{\rho}_{1}^{* m}$ and $\bar{\rho}_{2}^{* m}$ are bounded away from zero and infinity, $n / n_{m}=O(1)$, and $|S| \ll n$. Under Conditions 1-3, $\left\|\check{\boldsymbol{\beta}}_{S_{m}}^{m}-\boldsymbol{\beta}_{S_{m}}^{m}{ }^{*}\right\|_{2}=O_{p}\left(\sqrt{\left|S_{m}\right| / n_{m}}\right)$ for $m=1, \ldots, M$.

\subsection{Composite penalization}

Consider the objective function

$L(\boldsymbol{\beta})=\frac{1}{2 n} \sum_{m=1}^{M}\left(\boldsymbol{Y}^{m}-\boldsymbol{X}^{m} \boldsymbol{\beta}^{m}\right)^{\top} W_{m}\left(\boldsymbol{Y}^{m}-\boldsymbol{X}^{m} \boldsymbol{\beta}^{m}\right)+\sum_{j=1}^{p} p_{O, \lambda_{O}}\left(\sum_{m=1}^{M} p_{I, \lambda_{I}}\left(\left|\beta_{j}^{m}\right|\right)\right)$,

where the outer penalty $p_{O, \lambda_{O}}(\cdot)$ determines the overall importance of a variable, and the inner penalty $p_{I, \lambda_{I}}(\cdot)$ determines its individual importance. $\lambda_{O}$ and $\lambda_{I}$ 
are tuning parameters. A specific example is the composite MCP (cMCP) where both $p_{O, \lambda_{O}}$ and $p_{I, \lambda_{I}}$ are MCP.

Condition 4. $\theta_{O}=\inf \left\{\frac{|t|}{\lambda_{O}}: \frac{p_{O, \lambda_{O}}^{\prime}(|t|)}{\lambda_{O}}=0\right\}$ and $\theta_{I}=\inf \left\{\frac{|t|}{\lambda_{I}}: \frac{p_{I, \lambda_{I}}^{\prime}(|t|)}{\lambda_{I}}=0\right\}$ are bounded.

Take $J^{-m}=\max \left\{\sum_{i \neq m}^{M} I\left(\beta_{j}^{i} \neq 0\right), j \in S-S_{m}\right\}$ and $f_{I}^{\max }=\max _{t} p_{I, \lambda_{I}}(t)$.

Theorem 4. Assume Conditions $1-2$ and 4 . If

$$
C_{m}^{\dagger} \leq \frac{\min _{(j, m) \in \mathcal{A}}\left|\beta_{j}^{m *}\right|}{2} \sqrt{\frac{n_{m}}{\left|S_{m}\right|}}, \lambda_{I}<\frac{\min _{(j, m) \in \mathcal{A}}\left|\beta_{j}^{m *}\right|}{2 \theta_{I}}, \lambda_{O} \theta_{O}>f_{I}^{\max } \max _{m}\left(J^{-m}\right),
$$

then $\check{\boldsymbol{\beta}}$ is a local minimizer of $(\mathbb{4 . 2})$ with probability at least $1-\tau_{2}$, where

$$
\begin{aligned}
\tau_{2}= & \sum_{m=1}^{M} \exp \left\{-C_{m}^{\dagger 2} \frac{\left|S_{m}\right|\left(\underline{\rho}_{1}^{* m}\right)^{2}}{8 \bar{\rho}_{2}^{* m} \sigma_{m}^{2}}\right\}+2|S| \sum_{m=1}^{M} \exp \left\{-\frac{n^{2} p_{I, \lambda_{I}}^{2}(0+) p_{O, \lambda_{O}}^{\prime 2}\left(J^{-m} f_{I}^{\max }\right)}{2 n_{m} \bar{\rho}_{2}^{* m} \sigma_{m}^{2}\left(1+\psi_{m}^{*}\right)^{2}}\right\} \\
& +2(p-|S|) \sum_{m=1}^{M} \exp \left\{-\frac{n^{2} p_{I, \lambda_{I}}^{\prime 2}(0+) p_{O, \lambda_{O}}^{\prime 2}(0+)}{2 n_{m} \Lambda_{m} \sigma_{m}^{2}\left(1+\psi_{m}^{*}\right)^{2}}\right\} .
\end{aligned}
$$

Corollary 4. Suppose that for $m=1, \ldots, M, \rho_{1}^{* m}, \bar{\rho}_{2}^{* m}$, and $\Lambda_{m}$ are bounded away from zero and infinity, $n / n_{m}=O(1),|\bar{S}| \ll n, \log p=O\left(n^{\alpha}\right)$ with $\alpha<1$, and $\psi_{m}^{*}=O\left(n^{\alpha_{1}}\right)$ with $\alpha_{1} \in[0,1 / 2)$. Under Conditions 1,2 and 4 , if $\lambda_{I}<\min _{(j, m) \in \mathcal{A}}\left|\beta_{j}^{m *}\right| / 2 \theta_{I}, \lambda_{O} \theta_{O}=M f_{I}^{\max }$, and $\lambda_{I} \lambda_{O} \gg n^{(\alpha-1) / 2+\alpha_{1}}$, composite penalization can achieve two-level selection consistency.

Remark 4. Liu, Huang and Ma (2014) also suggests the composition of MCP and LASSO. We conjuncture that it is estimation consistent, and can consistently identify the overall importance of variables, but is not consistent at the individual level in general.

\subsection{Sparse group penalization}

Consider the objective function

$$
\begin{aligned}
L(\boldsymbol{\beta})= & \frac{1}{2 n} \sum_{m=1}^{M}\left(\boldsymbol{Y}^{m}-\boldsymbol{X}^{m} \boldsymbol{\beta}^{m}\right)^{\top} W_{m}\left(\boldsymbol{Y}^{m}-\boldsymbol{X}^{m} \boldsymbol{\beta}^{m}\right)+\sum_{j=1}^{p} p_{1, \lambda_{1}}\left(\left\|\boldsymbol{\beta}_{j}\right\|_{2}\right) \\
& +\sum_{j=1}^{p} \sum_{m=1}^{M} p_{2, \lambda_{2}}\left(\left|\beta_{j}^{m}\right|\right) .
\end{aligned}
$$

$\lambda_{1}$ and $\lambda_{2}$ are tuning parameters. Here the penalty is the sum of group and individual penalties. The first penalty determines the overall importance of a variable, and the second penalty determines its individual importance. 
Consider penalties $p_{1, \lambda_{1}}$ and $p_{2, \lambda_{2}}$ that satisfy Conditions 2 and 4 with bounded constants $\theta_{1}$ and $\theta_{2}$.

Theorem 5. Suppose that Conditions 1-2 and 4 hold. If

$$
C_{m}^{\dagger} \leq \frac{\min _{(j, m) \in \mathcal{A}}\left|\beta_{j}^{m *}\right|}{2} \sqrt{\frac{n_{m}}{\left|S_{m}\right|}}, \quad \lambda_{1}<\frac{\min _{j \in S}\left\|\boldsymbol{\beta}_{j}{ }^{*}\right\|_{2}}{2 \theta_{1}}, \lambda_{2}<\frac{\min _{(j, m) \in \mathcal{A}}\left|\beta_{j}^{m *}\right|}{2 \theta_{2}},
$$

then $\check{\boldsymbol{\beta}}$ is a local minimizer of $(4.3)$ with probability at least $1-\tau_{3}$, where

$$
\begin{aligned}
\tau_{3}= & \sum_{m=1}^{M} \exp \left\{-C_{m}^{\dagger 2} \frac{\left|S_{m}\right|\left(\underline{\rho}_{1}^{* m}\right)^{2}}{8 \bar{\rho}_{2}^{* m} \sigma_{m}^{2}}\right\}+2|S| \sum_{m=1}^{M} \exp \left\{-\frac{n^{2} p_{2, \lambda_{2}}^{\prime 2}(0+)}{2 n_{m} \bar{\rho}_{2}^{* m} \sigma_{m}^{2}\left(1+\psi_{m}^{*}\right)^{2}}\right\} \\
& +2(p-|S|) \sum_{m=1}^{M} \exp \left\{-\frac{n^{2}\left[p_{1, \lambda_{1}}^{\prime}(0+)+p_{2, \lambda_{2}}^{\prime}(0+)\right]^{2}}{2 n_{m} \Lambda_{m} \sigma_{m}^{2}\left(1+\psi_{m}^{*}\right)^{2}}\right\} .
\end{aligned}
$$

Thus the sparse group penalization also enjoys the consistency properties. For theoretical purposes, $p_{1, \lambda_{1}}$ and $p_{2, \lambda_{2}}$ do not need to take the same form, but using the same $p_{1, \lambda_{1}}$ and $p_{2, \lambda_{2}}$ may facilitate computation.

Corollary 5. Suppose that for $m=1, \ldots, M, \underline{\rho}_{1}^{* m}, \bar{\rho}_{2}^{* m}$, and $\Lambda_{m}$ are bounded away from zero and infinity, $n / n_{m}=O(1),|S| \ll n, \log p=O\left(n^{\alpha}\right)$ with $\alpha<1$, and $\psi_{m}^{*}=O\left(n^{\alpha_{1}}\right)$ with $\alpha_{1} \in[0,1 / 2)$. Under Conditions $1-2$ and 4 , if $\lambda_{1}<$ $\min _{j \in S}\left\|\boldsymbol{\beta}_{j}{ }^{*}\right\|_{2} / 2 \theta_{1}, \lambda_{2}<\min _{(j, m) \in \mathcal{A}}\left|\beta_{j}^{m *}\right| / 2 \theta_{2}, \lambda_{1} \gg n^{-1 / 2+\alpha_{1}}$ and $\lambda_{1}+\lambda_{2} \gg$ $n^{(\alpha-1) / 2+\alpha_{1}}$, sparse group penalization achieves two-level selection consistency.

\section{Numerical Study}

\subsection{Computation}

With the weighted LS approach, the loss function (2.2) has a least squares form. In single-dataset analysis with a LS loss, several computational algorithms have been developed for group penalization, composite penalization, and sparse group penalization (Friedman, Hastie, and Tibshirani (2010); Breheny and Huang (2009); Liu, Huang and Ma (2014)). Here we adopt the existing gradient descent algorithms with minor modifications. Convergence properties can be derived following Breheny and Huang (201) and references therein. Details are omitted here. The penalization methods involve the tuning parameter $\lambda\left(\lambda_{I}, \lambda_{O}, \lambda_{1}, \lambda_{2}\right)$ and the theorems provide results on the asymptotic order. MCP also involves an additional regularization parameter $a$. We consider a small number of values for $a$, in particular including $1.8,3,6$, and 10. In numerical study, we use 5-fold cross validation for tuning parameter selection. 


\subsection{Simulation}

We simulated three datasets, each with 100 subjects. For each subject, we simulated 1,000 covariates with a joint normal distribution, having marginal means equal to zero and variances equal to one. Consider two correlation structures, the first of which is the auto-regressive (AR) correlation, where covariates $j$ and $k$ have correlation coefficient $\rho^{|j-k|} . \rho=0.2,0.5$, and 0.8 , corresponding to weak, moderate, and strong correlations, respectively. The second is the banded correlation. Here three scenarios were considered: in the first scenario, covariates $j$ and $k$ have correlation coefficient 0.3 if $|j-k|=1$ and 0 otherwise; in the second, covariates $j$ and $k$ have correlation coefficient 0.6 if $|j-k|=1,0.3$ if $|j-k|=2$, and 0 otherwise; in the third, covariates $j$ and $k$ have correlation coefficient 0.6 if $|j-k|=1,0.3$ if $|j-k|=2,0.15$ if $|j-k|=3$, and 0 otherwise. Both homogeneity and heterogeneity models were simulated. Under the homogeneity model, all three datasets had the same twenty important covariates; under the heterogeneity model, each dataset had twenty important covariates. The three datasets shared ten important covariates in common, and the rest important covariates were dataset-specific. Under both models, there were a total of sixty true positives. The nonzero coefficients were randomly generated from a normal with mean zero and variance 0.3125 and 1.25 , representing low and high signal levels. The log event times were generated from the AFT models with intercept 0.5 and $\mathrm{N}(0,1)$ random errors. The log censoring times were independently generated from uniforms. The overall censoring rate was about $30 \%$.

The simulated data were analyzed using group MCP (GMCP), composite MCP (cMCP), and sparse group MCP (SGMCP). We also considered two alternatives. The first was a meta-analysis method, where each dataset was analyzed separately using MCP, and then the analysis results were combined across datasets. The second was a pooled analysis method, where the three datasets were combined into a big data matrix, and then variable selection was conducted using MCP. The differences across simulated datasets were smaller than those encountered in practice, which favors meta- and pooled analysis. We acknowledge that multiple other methods are applicable to the simulated data. The two alternatives have the closest framework to the proposed methods.

Summary results based on 200 replicates are shown in Table 1 and 2. Performance of the integrative analysis methods, as well as the alternatives, depend on the similarity of sparsity structures across datasets, correlation structure, and signal level. As an example of the homogeneity model, consider the correlation structure "Banded 2" in Table 1. The homogeneity model favors GMCP, which identifies 34.7 true positives with an average model size 45.2. The cMCP method identifies fewer true positives (30.5), which a large number of false positives are 
Table 1. Simulation at the low signal level. In each cell, the first row is the number of true positives (sd), and the second row is model size (sd).

\begin{tabular}{|ccccrc|}
\hline Correlation & Meta & Pooled & GMCP & cMCP & SGMCP \\
\hline \multirow{3}{*}{ AR $\rho=0.2$} & $30.3(5.7)$ & $29.0(8.4)$ & $48.8(6.2)$ & $42.6(4.2)$ & $36.5(6.7)$ \\
& $62.4(19.1)$ & $56.5(29.3)$ & $57.4(9.4)$ & $193.2(13.9)$ & $39.1(8.3)$ \\
\hline \multirow{2}{*}{ AR $\rho=0.5$} & $20.4(6.0)$ & $18.3(6.7)$ & $39.5(7.9)$ & $33.3(8.1)$ & $28.6(6.9)$ \\
& $38.7(17.7)$ & $31.2(16.3)$ & $50.8(12.4)$ & $160.6(83.0)$ & $30.9(9.1)$ \\
\hline \multirow{2}{*}{ AR $\rho=0.8$} & $10.9(2.6)$ & $10.3(3.3)$ & $24.8(7.7)$ & $18.3(4.1)$ & $16.8(5.2)$ \\
& $17.9(6.1)$ & $15.5(6.4)$ & $34.4(12.8)$ & $75.4(59.2)$ & $18.6(7.2)$ \\
\hline \multirow{2}{*}{ Banded 1 } & $26.7(5.8)$ & $25.1(7.6)$ & $46.2(7.6)$ & $40.3(4.5)$ & $34.7(6.2)$ \\
& $54.3(18.7)$ & $48.7(26.1)$ & $56.5(12.6)$ & $196.6(12.7)$ & $37.8(8.9)$ \\
\hline \multirow{2}{*}{ Banded 2 } & $17.6(4.5)$ & $16.1(5.0)$ & $34.7(8.3)$ & $30.5(6.0)$ & $25.6(5.9)$ \\
& $30.4(11.6)$ & $25.4(12.5)$ & $45.2(13.7)$ & $149.7(95.0)$ & $27.4(7.2)$ \\
\hline \multirow{2}{*}{ Banded 3 } & $17.7(5.3)$ & $16.2(4.9)$ & $37.3(7.3)$ & $31.4(5.8)$ & $26.1(6.3)$ \\
& $32.1(18.6)$ & $26.8(12.9)$ & $51.1(13.7)$ & $166.3(81.7)$ & $28.2(7.6)$ \\
\hline \multirow{2}{*}{ AR $\rho=0.2$} & $21.3(5.1)$ & $20.2(5.7)$ & $26.0(9.0)$ & $37.6(5.2)$ & $22.5(7.2)$ \\
& $35.5(13.8)$ & $31.4(13.9)$ & $53.0(20.3)$ & $199.2(40.3)$ & $28.4(11.0)$ \\
\hline \multirow{2}{*}{ AR $\rho=0.5$} & $16.8(5.1)$ & $16.7(5.3)$ & $22.8(6.2)$ & $31.7(r 6.9)$ & $18.8(5.7)$ \\
& $28.5(10.8)$ & $27.3(12.0)$ & $45.5(15.2)$ & $154.8(94.4)$ & $21.9(7.7)$ \\
\hline \multirow{2}{*}{ AR $\rho=0.8$} & $10.6(3.8)$ & $10.3(3.5)$ & $15.2(5.5)$ & $20.0(r 4.9)$ & $11.9(4.2)$ \\
& $17.0(6.3)$ & $15.3(6.3)$ & $31.4(12.9)$ & $99.9(84.4)$ & $15.3(6.8)$ \\
\hline \multirow{2}{*}{ Banded 1 } & $20.4(4.8)$ & $19.9(6.0)$ & $25.2(6.7)$ & $35.3(6.7)$ & $20.9(6.0)$ \\
& $35.2(15.2)$ & $31.3(13.9)$ & $48.9(14.5)$ & $172.2(77.9)$ & $24.9(7.9)$ \\
\hline \multirow{2}{*}{ Banded 2 } & $16.1(4.0)$ & $15.1(3.9)$ & $21.4(6.1)$ & $28.0(5.4)$ & $17.5(4.8)$ \\
& $24.9(8.4)$ & $22.8(7.7)$ & $44.0(12.2)$ & $129.9(103.4)$ & $21.0(6.2)$ \\
\hline \multirow{2}{*}{ Banded 3 } & $15.9(3.6)$ & $15.2(4.4)$ & $20.2(6.0)$ & $27.1(6.2)$ & $17.8(4.9)$ \\
& $26.8(10.8)$ & $24.3(10.2)$ & $43.3(14.2)$ & $102.7(115.7)$ & $22.3(7.5)$ \\
\hline
\end{tabular}

identified, with an average model size 149.7. SGMCP identifies 25.6 true positives, with a very small number of false positives (average model size 27.4). The meta-analysis and pooled analysis identify many fewer true positives (17.6 and 16.1, respectively). As an example of the heterogeneity model, consider the correlation structure "AR $\rho=0.5$ " in Table 2. The cMCP method identifies the most true positives (42.1 on average), but at the price of a large number of false positives (average model size 185.1). GMCP identifies 34.6 true positives and, by forcing the same sparsity structure across datasets, it also identifies a considerable number of false positives (average model size 61.0). SGMCP identifies 26.9 true positives with an average model size 30.2. The meta-analysis and pooled analysis methods identify fewer true positives. 
Table 2. Simulation at the high signal level. In each cell, the first row is the number of true positives (sd), and the second row is model size (sd).

\begin{tabular}{|ccrrrr|}
\hline Correlation & Meta & Pooled & GMCP & cMCP & SGMCP \\
\hline \multirow{4}{*}{ AR $\rho=0.2$} & $39.4(4.5)$ & $39.2(5 . \overline{4})$ & $58.3(2.3)$ & $52.3(2.9)$ & $49.9(3.8)$ \\
& $49.9(9.3)$ & $48.8(11.4)$ & $60.1(4.2)$ & $174.6(11.6)$ & $50.1(4.1)$ \\
\hline \multirow{2}{*}{ AR $\rho=0.5$} & $30.1(5.0)$ & $30.0(6.0)$ & $55.4(3.6)$ & $46.5(3.3)$ & $44.2(4.0)$ \\
& $42.0(10.1)$ & $41.8(12.3)$ & $58.3(4.3)$ & $179.8(15.3)$ & $44.5(4.2)$ \\
\hline \multirow{2}{*}{ AR $\rho=0.8$} & $17.4(3.8)$ & $17.1(3.9)$ & $46.5(6.7)$ & $29.5(6.4)$ & $29.6(5.9)$ \\
& $24.2(6.5)$ & $23.6(7.3)$ & $54.1(10.6)$ & $103.8(97.8)$ & $30.7(6.1)$ \\
\hline \multirow{2}{*}{ Banded 1 } & $36.9(4.7)$ & $35.9(5.1)$ & $57.2(2.7)$ & $50.3(2.9)$ & $47.9(4.3)$ \\
& $47.3(8.4)$ & $43.7(7.6)$ & $58.7(4.4)$ & $178.4(12.1)$ & $48.3(4.4)$ \\
\hline \multirow{2}{*}{ Banded 2 } & $25.9(4.3)$ & $25.5(4.7)$ & $53.3(4.6)$ & $41.1(3.4)$ & $38.6(5.5)$ \\
& $36.3(8.8)$ & $34.4(9.1)$ & $57.8(8.3)$ & $186.2(16.7)$ & $39.7(6.1)$ \\
\hline \multirow{2}{*}{ Banded 3 } & $27.1(3.8)$ & $26.5(4.3)$ & $53.7(4.5)$ & $42.4(4.4)$ & $40.8(4.7)$ \\
& $37.3(8.4)$ & $35.8(8.3)$ & $57.8(7.0)$ & $179.8(21.4)$ & $42.0(5.6)$ \\
\hline \multirow{3}{*}{ AR $\rho=0.2$} & $34.4(4.1)$ & $34.0(4.1)$ & $40.0(4.2)$ & $48.91(3.2)$ & $33.9(4.6)$ \\
& $39.7(6.0)$ & $37.9(4.8)$ & $69.2(7.9)$ & $180.4(18.9)$ & $36.6(4.7)$ \\
\hline \multirow{2}{*}{ AR $\rho=0.5$} & $25.9(4.5)$ & $24.1(5.9)$ & $34.6(5.7)$ & $42.1(4.1)$ & $26.9(4.8)$ \\
& $32.7(6.6)$ & $29.5(7.3)$ & $61.0(9.8)$ & $185.1(18.0)$ & $30.2(6.2)$ \\
\hline \multirow{2}{*}{ AR $\rho=0.8$} & $16.4(3.4)$ & $15.6(3.5)$ & $23.7(5.6)$ & $26.8(5.3)$ & $17.5(4.4)$ \\
& $22.2(5.1)$ & $21.3(6.5)$ & $44.3(10.3)$ & $157.5(87.3)$ & $20.9(5.6)$ \\
\hline \multirow{2}{*}{ Banded 1 } & $30.8(4.1)$ & $30.2(4.6)$ & $36.8(5.3)$ & $45.8(3.1)$ & $30.0(5.2)$ \\
& $36.0(5.8)$ & $35.4(6.7)$ & $64.1(9.3)$ & $177.7(17.3)$ & $32.6(6.7)$ \\
\hline \multirow{2}{*}{ Banded 2 } & $22.9(4.6)$ & $22.4(4.1)$ & $32.1(5.9)$ & $36.6(4.3)$ & $25.2(4.9)$ \\
& $29.3(7.8)$ & $27.5(5.4)$ & $57.4(8.4)$ & $169.2(51.2)$ & $28.6(5.3)$ \\
\hline \multirow{2}{*}{ Banded 3 } & $23.0(4.6)$ & $22.6(4.2)$ & $31.6(6.2)$ & $37.4(5.0)$ & $24.2(6.8)$ \\
& $28.7(5.8)$ & $27.9(5.3)$ & $57.1(9.9)$ & $169.2(42.1)$ & $26.6(7.5)$ \\
\hline
\end{tabular}

\subsection{Analysis of lung cancer prognosis data}

In the U.S., lung cancer is the most common cause of cancer death for both men and women. To identify genetic markers associated with the prognosis of lung cancer, gene profiling studies have been extensively conducted. We follow Xie et al. (201]) and collect data from four independent studies with gene expression measurements. The UM (University of Michigan Cancer Center) dataset has a total of 92 patients, with 48 deaths during follow-up. The median follow-up is 55 months. The HLM (Moffitt Cancer Center) dataset has a total of 79 patients, with 60 deaths during follow-up. The median follow-up is 39 months. The DFCI (Dana-Farber Cancer Institute) dataset has a total of 78 patients, with 35 deaths during follow-up. The median follow-up is 51 months. The MSKCC dataset has a total of 102 patients, with 38 deaths during follow-up. The median follow-up is 43.5 months. 
Table 3. Analysis of lung cancer data using cMCP: identified genes and their estimates.

\begin{tabular}{|c|c|c|c|c|c|}
\hline Probe & Gene & UM & HLM & DFCI & MSKCC \\
\hline 201462_at & SCRN1 & & 0.0045 & & \\
\hline 202637_s_at & ICAM1 & & & & 0.0037 \\
\hline 203240_at & FCGBP & & 0.0024 & & \\
\hline 203876_s_at & MMP11 & & & & -0.0013 \\
\hline 203917_at & CXADR & & & & 0.0040 \\
\hline 203921_at & CHST2 & 0.0024 & & & \\
\hline 204855_at & SERPINB5 & -0.0008 & & & \\
\hline 205234_at & SLC16A4 & & & & -0.0016 \\
\hline 205399_at & DCLK1 & & & & -0.0031 \\
\hline 206461_x_at & MT1H & & & -0.0008 & \\
\hline 206754_s_at & CYP2B6 & & & 0.0048 & \\
\hline 206994_at & CST4 & & -0.0017 & & \\
\hline 207850_at & CXCL3 & & -0.0155 & & \\
\hline 208025_s_at & HMGA2 & & & -0.0016 & \\
\hline 208451_s_at & $\mathrm{C} 4 \mathrm{~A}$ & & 0.0038 & & \\
\hline 208607_s_at & SAA2 & & & & 0.0044 \\
\hline 209343_at & EFHD1 & & & & 0.0028 \\
\hline 212328_at & LIMCH1 & & & 0.0028 & \\
\hline 212338_at & MYO1D & & 0.0019 & & \\
\hline 213338_at & TMEM158 & & & -0.0003 & \\
\hline 214452_at & BCAT1 & & & & 0.0004 \\
\hline 215867_x_at & CA12 & & -0.0054 & & \\
\hline 218677_at & S100A14 & & & & -0.0081 \\
\hline 219654_at & PTPLA & & -0.0109 & & \\
\hline 219747_at & NDNF & 0.0001 & & & \\
\hline 220952_s_at & PLEKHA5 & & -0.0018 & & \\
\hline 221841_s_at & KLF4 & -0.0024 & & & \\
\hline 222043_at & CLU & & 0.0008 & & \\
\hline
\end{tabular}

Gene expressions were measured using Affymetrix U122 plus 2.0 arrays. A total of 22,283 probe sets were profiled in the four datasets. We first conducted gene expression normalization for each dataset separately, and normalization across datasets was also conducted to enhance comparability. To further remove noise and improve stability, we conducted a marginal screening and kept the top 2,000 genes for downstream analysis. The expression of each gene in each dataset was normalized to have zero mean and unit variance.

We analyzed data using cMCP (Table 3), SGMCP (Table S2.1), metaanalysis (Table S2.2), pooled analysis (Table S2.3), and GMCP (Table S2.4). Although there is overlap, different methods identified significantly different sets of genes. The cMCP method identifies more genes, particularly many more than SGMCP. Such a result fits the pattern observed in simulation, but here we are 
not able to objectively evaluate the marker selection results. To provide further insights, we evaluated prediction performance using a cross-validation based approach. We split the samples into a training and a testing set with size 3:1. Estimates were generated using the training set samples and used to make prediction for the testing set samples. We separated the testing set samples into two sets with equal sizes based on the $\boldsymbol{X}^{m} \boldsymbol{\beta}^{m}$ 's. The logrank statistic was computed, evaluating survival difference of the two sets. To reduce the risk of an extreme split, we repeated this process 100 times and computed the average logrank statistics as 7.65 (cMCP), 4.95 (SGMCP), 5.35 (meta-analysis), 5.2 (pooled analysis), and 6.45 (GMCP). All methods were able to separate samples into sets with different survival risk. The cMCP method had the best prediction performance (p-value 0.0057).

\section{Discussion}

We have studied the integrative analysis of survival data under the AFT model. Existing research on this topic has been scattered, and this is the first systematic study of this complicated problem. Both homogeneity and heterogeneity models have been considered, along with multiple penalization methods. As against existing studies, the present study rigorously establishes the selection and estimation consistency properties. Although some theoretical development was motivated by existing studies, the heterogeneity across multiple datasets and specific data and model settings were new. Especially, the properties of sparse group penalization have not been studied in single-dataset analysis. The computational aspect here is similar to that in the literature and is largely omitted. Tuning parameter selection using cross validation shows reasonable performance in simulation and data analysis. Theoretical investigation of the consistency of cross validation is challenging, and postponed. Our study directly compares different methods. The advantage of GMCP under the homogeneity model is expected. Under the heterogeneity model, cMCP may identify a few more true positives, but at the price of a large number of false positives. More studies on finite sample properties are needed. In simulation, a total of 24 settings were considered and they showed similar patterns. In data analysis, different methods identify different sets of genes. The observed patterns are similar to those in simulation. The cMCP identifies the most genes and also has the best prediction performance. More extensive, especially biological studies may be needed to fully comprehend the data analysis results. We have focused on survival data and the AFT model. Extensions to other data and models are of interest in future study.

\section{Supplementary Materials}

The online supplementary material contains proofs and additional numerical results. 


\section{Acknowledgements}

We thank the Editor and reviewer for their careful review and insightful comments. This study was partly supported by National Natural Science Foundation of China (11401561, 71471152, 71201139, 71301162), National Social Science Foundation of China (13\&ZD148, 13CTJ001), and NIH (R03CA182984, P30CA016359, R01CA142774).

\section{References}

Breheny, P. and Huang, J. (2009). Penalized methods for bi-level variable selection. Statist. Interface 2, 369-380.

Breheny, P. and Huang, J. (2011). Coordinate descent algorithms for nonconvex penalized regression, with applications to biological feature selection. Ann. Appl. Statist. 5, 232-253.

Bühlmann, P. and van de Geer, S. (2011). Statistics for High-Dimensional Data: Methods, Theory and Applications. Springer.

Fan, J. and Li, R. (2001). Variable selection via nonconcave penalized likelihood and its oracle properties. J. Amer. Statist. Assoc. 96, 1348-1360.

Fan, J. and Lv, J. (2011). Nonconcave penalized likelihood with NP-dimensionality. IEEE Trans. Inform. Theory 57, 5467-5484.

Friedman, J., Hastie, T. and Tibshirani, R. (2010). A note on the group lasso and a sparse group lasso. Technical Report, Stanford University.

Guerra, R. and Goldstein, D. R. (2009). Meta-Analysis and Combining Information in Genetics and Genomics. Chapman and Hall/CRC.

Huang, J., Breheny, P. and Ma, S. (2012), A selective review of group selection in highdimensional models. Statist. Sci. 27, 481-499.

Liu, J., Huang, J., Xie, Y. and Ma, S. (2013). Sparse group penalized integrative analysis of multiple cancer prognosis datasets. Genetics Res. 95, 68-77.

Liu, J., Huang, J. and Ma, S. (2014). Integrative analysis of cancer diagnosis studies with composite penalization. Scand. J. Statist. 41, 87-103.

Ma, S., Huang, J. and Song, X. (2011a). Integrative analysis and variable selection with multiple high-dimensional data sets. Biostatistics 12, 763-775.

Ma, S., Huang, J., Wei, F., Xie, Y. and Fang, K. (2011b). Integrative analysis of multiple cancer prognosis studies with gene expression measurements. Statist. Medicine 30, 3361-3371.

Ma, S., Dai, Y., Huang, J. and Xie, Y. (2012). Identification of breast cancer prognosis markers via integrative analysis. Comput. Statist. Data Anal. 56, 2718-2728.

Stute, W. (1993). Consistent estimation under random censorship when covariables are present. J. Multivariate Anal. 45, 89-103.

Xie, Y., Xiao, G., Coombes, K., Behrens, C., Solis, L., Raso, G., Girard, L., Erickson, H., Roth, J., Heymach, J., Moran, C., Danenberg, K., Minna, J. and Wistuba, I. (2011). Robust gene expression signature from formalin-fixed paraffin-embedded samples predicts prognosis of non-small-cell lung cancer patients. Clin Cancer Res. 17, 5705-5714.

Ying, Z. (1993). A large sample study of rank estimation for censored regression data. Ann. Statist. 21, 76-99.

Zhang, C.-H. (2010). Nearly unbiased variable selection under minimax concave penalty. Ann. Statist. 38, 894-942. 
Zhao, P. and Yu, B. (2006). On model selection consistency of Lasso. J. Mach. Learn. Res. 7, 2541-2563.

School of Mathematical Sciences, University of Chinese Academy of Sciences, No. 19A Yuquan Road, Beijing, 100049, China.

E-mail: qzzhang@ucas.ac.cn

School of Mathematical Sciences, University of Chinese Academy of Sciences, No. 19A Yuquan Road, Beijing, 100049, China.

E-mail: sgzhang@ucas.ac.cn

Center of Quantitative Medicine, Duke-NUS School of Medicine, Singapore.

E-mail: jin.liu@duke-nus.edu.sg

Department of Statistics and Actuarial Science, University of Iowa, Chicago, IL 60612, USA.

E-mail: jian-huang@uiowa.edu

Department of Biostatistics, Yale University, New Haven, CT 06520-8034, USA.

E-mail: shuangge.ma@yale.edu

(Received June 2014; accepted May 2015) 